\title{
La influencia del apego y el autoconcepto en los problemas de comportamiento de los niños y niñas de familias en desventaja socioeconómica
}

\section{The influence of attachment and self-concept on behavior problems in children from socioeconomically disadvantaged families}

\author{
Alezandra Torres \\ amerindia492@gmail.com \\ M. ${ }^{a}$ José Rodrigo \\ mjrodri@ull.es \\ Universidad de la Laguna, España
}

\section{Resumen:}

Este artículo analiza la relación entre el apego, el autoconcepto y los problemas de comportamiento en niños/as de 6 a 12 años, provenientes de familias en riesgo psicosocial medio, caracterizadas por situaciones de desventaja socioeconómica $(\mathrm{N}=102)$ y de familias normalizadas $(\mathrm{N}=$ 114) de la isla de Tenerife (España). Todos asistían a un programa de intervención llevado a cabo en ludotecas o en colegios. Los participantes produjeron narrativas sobre las figuras de apego, valoraron su autoconcepto en diferentes ámbitos, y finalmente sus educadores evaluaron la presencia de problemas de comportamiento de tipo internalizado y externalizado. Los resultados muestran que las dificultades en las relaciones de apego y un menor autoconcepto se relacionan positivamente con problemas de comportamiento de tipo externalizado así como con problemas cognitivos y sociales. Estas relaciones estuvieron moderadas por el estatus de riesgo familiar demostrando la

\begin{abstract}
:
This paper examines the relationship between attachment, self-concept and behavior problems in children aged 6 to 12 years old, from families at psychosocial risk, characterized by socioeconomic disadvantage $(\mathrm{N}=102)$ and standard families $(\mathrm{N}=114)$ of the island of Tenerife (Spain). All were attending an intervention program conducted in playgrounds or schools. The participants produced narratives about attachment figures, rated their self-concept in different areas, and finally their educators evaluated the presence of behavioral problems of internalized, externalized and cognitive-social type. The results show that the difficulties in attachment relationships and a lower self-concept are positively related to behavior problems such as externalizing and cognitive and social problems. These relationships varied according to family risk status demonstrating the increased vulnerability of children in families at risk when it comes to having behavior problems that can affect their development.
\end{abstract}


La influencia del apego y el autoconcepto en los problemas de comportamiento de los niños y niñas de familias en desventaja socioeconómica

AlezANDRA TORRES y M. ${ }^{a}$ José Rodrigo

mayor vulnerabilidad de los niños y niñas de familias en riesgo para presentar problemas de comportamiento que pueden afectar a su desarrollo.

\section{Palabras clave:}

Narrativas de apego, autoconcepto, problemas de comportamiento, externalización, menores, riesgo psicosocial, desventaja socioeconómica.

\section{Key words:}

Narratives of attachment, self-concept, behavior problems, externalization, minors, psychosocial risk, socioeconomical disadvantage.

\section{Résumé:}

Ce document examine la relation entre l'affection, le concept de soi et des problèmes de comportement chez les enfants 6-12 ans issus de familles à risques psychosociaux, caractérisées par des désavantages socio-économiques $(\mathrm{N}=102)$ et les familles standard $(\mathrm{N}$ =114) de l'île de Tenerife (Espagne). Tous ont assisté à un programme d'intervention mis en pratique dans des salles de jeux ou dans des écoles. Les participants ont produit des récits sur les figures d'affection, ont évalué leur concept de soi dans différents domaines, et, enfin, leurs éducateurs ont évalué la présence de problèmes de comportement intériorisé et de type extériorisé. Les résultats montrent que les difficultés dans les relations d'affection et une image de soi plus faible ont des problèmes de comportement de type intériorisé, cognitifs et sociaux. Ces relations ont été modérées par le statut de risque familial démontrant la vulnérabilité accrue des enfants dans les familles à risque d'avoir des problèmes de comportement qui peuvent affecter leur développement.

\section{Mots clés:}

Récits d'attachement, le concept de soi, problèmes de comportement, d'extériorisation, les enfants, risques psychosociaux, socio-économique défavorisé.

Fecha de recepción: 23-4-2013

Fecha de aceptación: 7-1-2014

\section{Agradecimientos}

Expresamos nuestro agradecimiento a los profesores y educadores de los equipos de riesgo de la isla de Tenerife por colaborar en la recogida de datos así como a los menores que participaron. Este estudio ha sido posible gracias al apoyo del Ministerio de Ciencia e Innovación mediante el proyecto (TRA2009_0145) concedido al segundo autor.

\section{Introducción}

El apego y el autoconcepto parecen ser dimensiones interpersonales claves para predecir el bienestar psicológico de los niños/as y los posibles 
problemas en su ajuste psicosocial. Esa noción de ajuste psicosocial es de gran valor en el escenario educativo porque su carencia puede llegar a afectar áreas determinantes en el desarrollo de competencias académicas, como es el área comunicativa y en concreto el lenguaje, el cual es considerado como piedra angular de muchos aspectos cognitivos y socioemocionales del desarrollo (Gleason y Ratner, 2008; Hoff, 2008), así como en otras áreas importantes como la referida a las relaciones sociales y a la regulación del comportamiento.

El análisis de la representación que se hacen los niños de sus figuras de apego ha servido para caracterizar algunos rasgos del perfil de ajuste psicosocial de éstos, sobre todo en la primera infancia, como pueden ser las dificultades en la regulación de las emociones (Shields, Ryan y Cicchetti, 2001) y la mayor vulnerabilidad ante los problemas de comportamiento (Sroufe, Carlson, Levy, y Egeland, 1999). El apego en la edad infantil ha sido definido como un vínculo emocional de larga duración que el niño/a forma con su cuidador (Bowlby, 1980). Los niño/as se sienten seguros en sus relaciones con las figuras de apego, cuando ellos las han percibido como accesibles, sensibles y atentas a sus necesidades. Los niños con relaciones de apego seguras cuentan con unos cuidadores que representan una base de seguridad sobre la cual explorar el mundo. Cuando, por el contrario, hay carencia de accesibilidad física y emocional por parte de los cuidadores, lo niños forman relaciones de apego inseguras y consecuentemente sus cuidadores ya no representan una base confiable desde la que explorar el mundo (Brumariu y Kerns, 2010; Weinfield, Sroufe, Egeland, y Carlson, 2008).

El autoconcepto, por su parte, es otro aspecto clave del desarrollo del yo para entender los problemas de ajuste psicosocial en los niños/ as, porque hace referencia a la percepción de sí mismo que se ha ido formando a través de las valoraciones reflejadas en las relaciones con los otros significativos, así como también a partir de comparaciones sociales y de atribuciones sobre si mismos (Harter, Waters y Whitesell, 1998). Sin embargo, a partir de los ocho años, los niños desarrollan sistemas de representación que les permiten integrar diferentes características de sí mismos y diferentes puntos de vista para hacer conceptualizaciones más complejas y articuladas. En cualquier caso, el desarrollo de un autoconcepto positivo va a estar relacionado, ente otros aspectos, con su grado de ajuste escolar (González-Pienda, et al., 2000) y en general con estilos de vida más positivos, especialmente en 
La influencia del apego y el autoconcepto en los problemas de comportamiento de los niños y niñas de familias en desventaja socioeconómica

Alezandra Torres y M. ${ }^{a}$ José Rodrigo

la adolescencia (Baumeister, Campbell, Krueger y Vohs, 2003; Garaigordobil, Durá, y Pérez, 2005).

Varios estudios han encontrado relación entre el tipo de apego temprano que el niño establece con sus cuidadores y los problemas de externalización e internalización en la primera y segunda infancia (NICHD Early Child Care Research Network, 2006; Pasco Fearon y Belsky, 2011). Se ha encontrado que los niños/as clasificados con apegos inseguros presentan niveles más altos de internalización y externalización, en comparación con los presentados por los niños/as clasificados con un apego seguro (Muris, Meesters, y Van den Berg, 2003). El síndrome de internalización se refiere a los problemas de retraimiento, ansiedad depresión y disforia, así como sentimientos de inferioridad, timidez, autoconciencia, hipersensibilidad y quejas somáticas. Por su parte, el síndrome de externalización se refiere a problemas caracterizados por comportamientos desafiantes, disruptivos, agresivos y antisociales, así como hiperactividad, problemas de atención y de autocontrol. (Acchenbach, Howel, Quay y Conners, 1991; Bornstein, Hahn y Suwalsky, 2013).

Se han obtenido, también relaciones entre el autoconcepto y la autoestima y los problemas de comportamiento de tipo externalizado (Baumeister et al., 2003). Específicamente algunos autores plantean que la agresividad y el comportamiento antisocial en los niños/as y adolescentes son una expresión de su baja autoestima (Donnellan, Trzesniewski, Robins, Moffitt y Caspi, 2005).

El presente trabajo, se centra en en niños/as en la segunda infancia y pre-adolescencia que viven en familias en riesgo psicosocial medio. Por familias en riesgo psicosocial se entiende aquellas en las que los responsables del cuidado, atención, y educación del menor por circunstancias personales y relacionales, así como por influencias adversas de su entorno, hacen dejación de sus funciones parentales o hacen un uso inadecuado de las mismas, comprometiendo el desarrollo personal y social del menor, sin llegar a un nivel que se justifique una medida de protección (Rodrigo, Máiquez, Martín y Byrne, 2008). De manera específica, los casos familiares de riesgo medio se caracterizan por una situación de desventaja socioeconómica en la que se puede presentar indicadores como desempleo o necesidad de recibir alguna ayuda social, así como la presencia de diferentes estresores psicosociales (e.g., problemas conyugales, problemas derivados de las condiciones de vivienda), sin llegarse a producir una acumulación tal de los mismos que desembo- 
que en una desorganización familiar significativa (Rodríguez, Camacho, Rodrigo, Martín y Máiquez, 2006).

Algunos estudios en el contexto internacional han encontrado que durante los años escolares y la adolescencia, los hijos/as de familias en situación de riesgo muestran mayores problemas de ajuste psicosocial que la población de familias más normalizadas (Wadsby, Svedin y Sydsjö, 2007) indicando que hay una relación entre una situación de desventaja socioeconómica y una mayor vulnerabilidad a desarrollar problemas conductuales y emocionales sobre todo en la primera infancia y la adolescencia (Compas, 2004).

En el contexto español, en un estudio reciente sobre el proceso evolutivo de chicos/as y adolescentes de familias en riesgo psicosocial, se encontraron mayores problemas de ajuste social (valorados tanto en aspectos relacionados con la internalización y la externalización) entre quienes procedían de contextos familiares en riesgo psicosocial (Jiménez, 2009)

En relación a la situación de desventaja socioeconómica que caracteriza la situación de riesgo medio familiar, se ha reportado que los niños en edad escolar de familias en situación de desventaja económica, han mostrado también una elevada tasa de problemas de internalización y externalización (Burchinal, Roberts, Zeisel, Hennon, y Hooper, 2006).

Hay suficientes evidencias para considerar que la carencia material y de cuidados parentales adecuados puede afectar el desarrollo de las habilidades académicas y comportamentales, así como al desarrollo a largo plazo (Heckman, 2006). El capital tanto económico como emocional con el que cuentan las familias es determinante y sirve como fuente para la adquisición durante la infancia de las habilidades sociales y de auto-regulación, lo cual va a influir directamente en su funcionamiento socioemocional y académico conforme el niño/a avanza en la infancia media e incluso en años posteriores (Hill y Morris, 2008). Por ello resulta de interés dar a conocer estudios de este tipo en entornos escolares, ya que es necesario que el profesorado se familiarice con estas problemáticas familiares de una parte de su alumnado y también para que valore la importancia de favorecer la comunicación y el intercambio con las familias como mecanismo para que una buena educación sea posible (García, Gomariz, Hernández y Parra, 2010).

El objetivo de este estudio es, por tanto, conocer si dos aspectos cruciales del desarrollo del yo como son el apego y el autoconcepto, deri- 
La influencia del apego y el autoconcepto en los problemas de comportamiento de los niños y niñas de familias en desventaja socioeconómica

Alezandra Torres y M. ${ }^{a}$ José Rodrigo

vados de las relaciones establecidas con los primeros cuidadores en la primera infancia, predicen problemas de comportamiento tanto de internalización como de externalización, en niños/as en la infancia media y pre-adolescencia. Asimismo esperamos conocer cómo se produce ese impacto en chico/as provenientes de familias en riesgo psicosocial medio con respecto a los niños/as provenientes de familias normalizadas.

Dadas las edades de los niños/as, para la evaluación del apego se analizó su producción de narraciones, las cuales ofrecen indicios sobre cómo se representan las relaciones con las figuras de apego. La producción de narrativas se considera una vía importante para acceder a dichas representaciones o modelos mentales internos en los niños mayores de 4 años (Oppenheim, 2003; Shields, Ryan y Cicchetti, 2001). Otros estudios que incluyeron las narrativas sobre las relaciones de apego han demostrado que el contenido y la manera en que los niños cuentan historias ofrece una ventana importante para conocer sus modelos internos de pensamiento, a los cuales es difícil de acceder (Futh, O'Connor, Matias, Green y Scott, 2008).

Por su parte, la evaluación del autoconcepto se basó en auto-informes sobre múltiples dominios del autoconcepto para apresar toda su complejidad. Este análisis permite conocer de forma articulada cómo se está produciendo el desarrollo del yo, el cual sufre una consolidación importante a estas edades (Bracken y Lamprecht, 2003). Los problemas de comportamiento por su parte fueron evaluados mediante escalas cumplimentadas de manera individual por sus educadores.

La hipótesis de este estudio por tanto es que las dificultades en las relaciones de apego y un menor autoconcepto se relacionen positivamente con problemas de comportamiento de tipo externalizado e internalizado así como con problemas cognitivos y sociales. Asimismo, se espera que la pertenencia a familias de riesgo o normalizadas influya también en dichos resultados, presentando un desarrollo del yo más apropiado los hijos que provienen de familias normalizadas.

\section{Método}

Para el desarrollo de este estudio se ha seguido un modelo de investigación de tipo descriptivo y correlacional, ya que la finalidad era conocer las relaciones entre un grupo de variables medidas por instrumentos es- 
pecíficos (apego, autoconcepto y problemas de comportamiento). Todo ello para conocer mediante modelos de regresión lineal jerárquica qué ámbitos del apego y del autoncepto predicen mejor los problemas de comportamiento en chico/as de 6 a 12 años provenientes de familias en situación de riesgo psicosocial medio en relación con aquellos provenientes de familias normalizadas.

\section{Participantes}

Se seleccionaron 216 chico/as entre 6 y 12 años. Un primer grupo de 102 chicos/as provenía de familias con un nivel medio de riesgo psicosocial referidas por los servicios sociales municipales. El segundo grupo, 114 chicos/as, procedía de familias "normalizadas". Toda la recogida de información se hizo entre los años 2006 y 2008. El acceso a ambos grupos fue posible gracias a la colaboración de una ONG local que desarrollaba programas de Preservación familiar para padres/madres y para los niño/as en las ludotecas de los barrios. En dichas actividades podían participar tanto los chico/as procedentes de las familias en riesgo psicosocial, como los chico/as provenientes de familias normalizadas que acudían para participar en actividades de refuerzo educativo. En todos los casos se trataba de familias con origen en la Comunidad Autónoma Canaria. Gracias al apoyo de los educadores de la ludoteca fue posible organizar dos grupos pareados que tuvieran características similares respecto a variables como sexo, edad y grado escolar (Tabla 1).

Tabla 1. Caracterización de los grupos normalizado y riesgo de acuerdo con las variables sociodemográficas

\begin{tabular}{|c|c|c|c|c|}
\hline $\begin{array}{l}\text { Categorías } \\
\text { Sociodemográficas }\end{array}$ & $\begin{array}{c}\text { Normalizado } \\
(\%)\end{array}$ & $\begin{array}{c}\text { Riesgo Medio } \\
(\%)\end{array}$ & $\chi^{2}(1)$ & $P$ \\
\hline Sexo: & & & 4.03 & 0.030 \\
\hline Chicos & 41.2 & 54.9 & & \\
\hline Chicas & 58.8 & 45.1 & & \\
\hline Edad: & & & 0.001 & 0.556 \\
\hline Pequeños (6 a 9) & 50 & 50 & & \\
\hline Mayores (10 a 13) & 50 & 50 & & \\
\hline
\end{tabular}


La influencia del apego y el autoconcepto en los problemas de comportamiento de los niños y niñas de familias en desventaja socioeconómica

Alezandra Torres y M. ${ }^{a}$ José Rodrigo

Las familias de riesgo de este estudio, fueron valoradas por los técnicos de los Servicios Sociales mediante un instrumento estandarizado para evaluar el nivel de riesgo de las familias que recogía información sobre: zona de residencia de la familia, nivel de estudios y situación laboral del padre y de la madre, presencia de ayudas económicas, organización familiar (Inestabilidad laboral, deficiencia de organización de la economía doméstica, vivienda en malas condiciones), historia personal y características del padre y la madre, características del microsistema familiar, pautas educativas de riesgo, presencia de redes de apoyo e historial de adaptación de los hijos (Rodríguez et al. 2006). Las familias catalogadas en riesgo medio presentaban los siguientes indicadores: Vivienda en malas condiciones de mantenimiento, equipamiento y orden; Desconocimiento de las necesidades emocionales y cognitivas de los hijos; Falta de higiene del hijo; Normas excesivamente rígidas e inconsistentes; Retraso escolar del hijo; Carencia de redes sociales de apoyo; y Relación de pareja conflictiva.

\section{Instrumentos}

A continuación, se presentan los 3 instrumentos utilizados para indagar sobre la percepción de las figuras de apego, el autoconcepto y los problemas de comportamiento.

\section{Prueba de narrativa de los menores sobre sus cuidadores}

Inspirada en el Rochester Parenting Stories de Shields, Ryan y Cicchetti, (2001). Esta prueba permite conocer las representaciones de los niño/as de 6 a 12 años sobre sus figuras de apego. En la adaptación realizada para este estudio se incluyeron 5 viñetas sobre situaciones familiares para facilitar la producción de narrativas sobre las figuras de apego. Dichas viñetas fueron: 1 . El niño/a ha tenido una pesadilla y tiene mucho miedo, 2. El niño/a llega triste a casa porque sus amigos se han burlado de él/ella. En estas dos viñetas se evalúa la necesidad de ayuda y consuelo en momentos de estrés. 3. En casa un día el niño/a recoge la mesa después de comer; en esta viñeta se evalúa la autonomía conductual para tomar iniciativas. 4. En casa un día el niño/a ve la televisión con un hermano y de repente empiezan a pelear porque cada uno quiere ver un canal diferente, en esta viñeta se evalúan las estrategias de resolución 
de conflictos. 5. El niño/a en casa, jugando con un amigo, de repente tropiezan y rompen un jarrón, en esta viñeta se evalúa la percepción de la norma y la disciplina parental. Durante la prueba se hicieron las siguientes preguntas a los niños después de ver cada una de las viñetas: ¿Ante esta situación qué hace tu madre? ¿ y qué hace tu padre? ¿ y tú qué haces? ¿Cómo te sientes, en esta situación? ¿Por qué? En la primera viñeta, además se añade la pregunta ¿A quién Ilamarías?

Debido al retraso escolar de los menores del grupo de riesgo y al hecho de que se debían grabar en audio las narraciones producidas por los menores, el pase de esta prueba estuvo a cargo de los educadores pertenecientes a la ONG, a quienes se les hizo una formación específica. La media del índice Kappa en todas las categorías (0.929) indicó un nivel alto de acuerdo entre los dos jueces que valoraron las narraciones.

La codificación de las respuestas se realizó de acuerdo a las siguientes categorías:

a. Contenido (escala de 0-4):

- Responsividad: El grado en el que los cuidadores están accesibles y reconocen las necesidades físicas y emocionales de los chicos/as. Ejemplo: "Les Ilamo y entonces viene mi madre o mi padre" "Mi madre me pregunta si pasa algo".

- Apoyo a la autonomía: Si los padres apoyan los intentos de los chicos/as de hacer las cosas por su propia cuenta. Ejemplo: "A veces ayudo a mi madre a recoger la mesa" "En casa me han enseñado a que después de comer debo recoger la mesa, al menos debo recoger mis platos"

- Coerción emocional: Cuando los padres inducen estados emocionales negativos en el niño. Ejemplo: "A veces me riñe mi madre y me dice que soy torpe"

"Mi madre me dice que soy un tonto cuando alguien me empuja en el colegio y yo no respondo".

- Coerción física: El grado en que los padres castigan físicamente para ejercer el control. Ejemplo: "Si rompo el jarrón de mi madre, me arrestan pero antes seguro me dan un bofetón!!"

b. Estructura de las narraciones (escala de 0-2):

- Resistencia: El grado en el que el niño evita contar la narración. Ejemplo: "Pues no se"

- Pobreza del discurso: Se refiere a la falta de elementos en las narraciones, a la ausencia de detalles y especialmente a la ausencia 
La influencia del apego y el autoconcepto en los problemas de comportamiento de los niños y niñas de familias en desventaja socioeconómica

AlezANDRA TORRES y M. ${ }^{a}$ José Rodrigo

de temas emocionales. Ejemplo: "Llamo a mi madre.." "Llamo a mi padre" "Nada más..." "La verdad es que no tengo ni idea lo que haría"

- Tangencialidad: Se refiere a aquellas producciones narrativas lejanamente asociadas con las viñetas. Ejemplo: "Porque mi hermana siempre quiere tener el mando y a mí nunca me lo dan" "Los niños son así.."

- Incoherencia: el grado en que las narraciones no presentan una organización lógico-causal. Ejemplo: "Mi madre viene a hablar al colegio!!! y yo se le digo al profesor que un niño se rió de mi"

\section{Escala de autoconcepto infantil}

De Villa y Auzmendi (1999). Esta prueba propone el análisis de 10 ámbitos del autoconcepto: Autonomía, seguridad, deporte, vida familiar, mundo escolar, relaciones sociales, aspectos emocionales, autovalía, aspectos físicos y sentimientos de posesión. Está formada por 34 viñetas, en las que se representa a un grupo de niños/as en situaciones cotidianas relativas a cada uno de los ámbitos mencionados. En los personajes se pueden reconocer situaciones representativas de un autoconcepto positivo o de uno negativo.

La tarea consiste en que los niños/as digan con cuál personaje de las viñetas se identifican, para ello deben señalar aquellas viñetas que se asemejan a su forma de actuar. De este modo se facilita el que los niños con diferente nivel lector puedan entender y responder esta prueba de autoinforme. Es importante tener en cuenta que debido al retraso escolar del grupo de chico/as de riesgo medio, el formato de este cuestionario basado en viñetas sobre situaciones familiares cotidianas, fue de gran ayuda.

La prueba la pasaron los educadores entrenados del programa que habían pasado también la prueba de narrativas. Para la calificación de la prueba la escala utilizada fue de 0 a 1, siendo 1 la elección que corresponde a un autoconcepto positivo. Cada ámbito del autoconcepto se relacionaba con las siguientes viñetas: Autonomía (No. de viñeta: 1 , $24)$, seguridad $(3,17,29,32)$, deporte $(7,10,31)$, vida familiar $(4,25$, 16), mundo escolar $(9,22,26)$, relaciones sociales $(2,5,8,12,18,19,20$ y $27)$, aspectos emocionales (13, 23,30 y 34), autovalía (14, 21), aspectos físicos $(6,11,28$ y 33) y sentimientos de posesión (15). 


\section{Child Behavior Checklist (CBCL)}

De Achenbach y Edelbrock (1983). Este cuestionario evalúa la presencia de problemas de comportamiento relacionados con una variedad de perfiles psicopatológicos. Está compuesto por 113 ítems, a partir de los cuales se obtienen ocho factores llamados síndromes de primer orden (comportamiento agresivo, problemas atencionales, delincuencia, problemas sociales, problemas de pensamiento, quejas somáticas, retraimiento, ansiedad-depresión) y tres de segundo orden llamados internalizantes (retraimiento, quejas somáticas y ansiedad-depresión), externalizantes (comportamiento delincuente y comportamiento agresivo) y mixtos (problemas sociales, problemas de pensamiento y problemas de atención) (Achenbach, 1993). En nuestro caso utilizamos los factores de segundo orden. Se utilizó esta versión que podía ser contestada por los educadores, tanto de los chicos/as del grupo de riesgo como de los del grupo normalizado, ya que estos educadores conocían sobradamente las características comportamentales de estos chicos/as. Para calificar la prueba se usó una escala de 0 a 2. Las bondades de esta escala (alta fiabilidad de las escalas y alta validez) son muy conocidas por ser la de mayor difusión internacional en este ámbito. http://www.aseba.or/

\section{Resultados}

En el siguiente apartado se presentan los resultados de los análisis estadísticos. De una parte las correlaciones (Correlación de Pearson) para conocer el grado de relación entre las variables analizadas. De otra parte, los análisis predictivos (regresión lineal jerárquica) que tuvieron como objetivo conocer el poder predictivo de las variables de narrativas del apego y autoconcepto sobre los problemas de comportamientos en chicos/as de familias en riesgo psicosocial medio y en los de familias normalizadas. Con la idea de profundizar en el impacto diferencial entre los alumnos provenientes de un tipo y otro de familias, se crearon variables mixtas, multiplicando los valores de las variables anteriores y el grupo familiar de procedencia (riesgo-normalizado) y se incluyeron figuras para ilustrar aquellas interacciones que resultaran significativas. 
La influencia del apego y el autoconcepto en los problemas de comportamiento de los niños y niñas de familias en desventaja socioeconómica

Alezandra Torres y M. ${ }^{a}$ José Rodrigo

\section{Correlaciones}

En primer lugar, en las correlaciones entre las variables del apego y las variables de problemas de comportamiento, en la Tabla 2 se observan las correlaciones negativas y positivas entre las variables responsividad y coerción emocional y la dimensión de problemas cognitivos y sociales. Esto quiere decir que cuando los cuidadores eran percibidos por los menores como atentos y responsivos, los problemas cognitivos y sociales eran menores. En cambio cuando los cuidadores eran percibidos como efectuando más coerción emocional, los problemas cognitivos y sociales se incrementaban. Finalmente, se encontraron correlaciones significativas entre la variable pobreza de las narraciones del apego y las dimensiones externalización y problemas cognitivos y sociales, lo que indica que la producción de narraciones con más ausencia de detalles emocionales se relaciona con la mayor presencia de problemas de comportamiento de tipo externalizado, así como también mayores problemas cognitivos y sociales. 


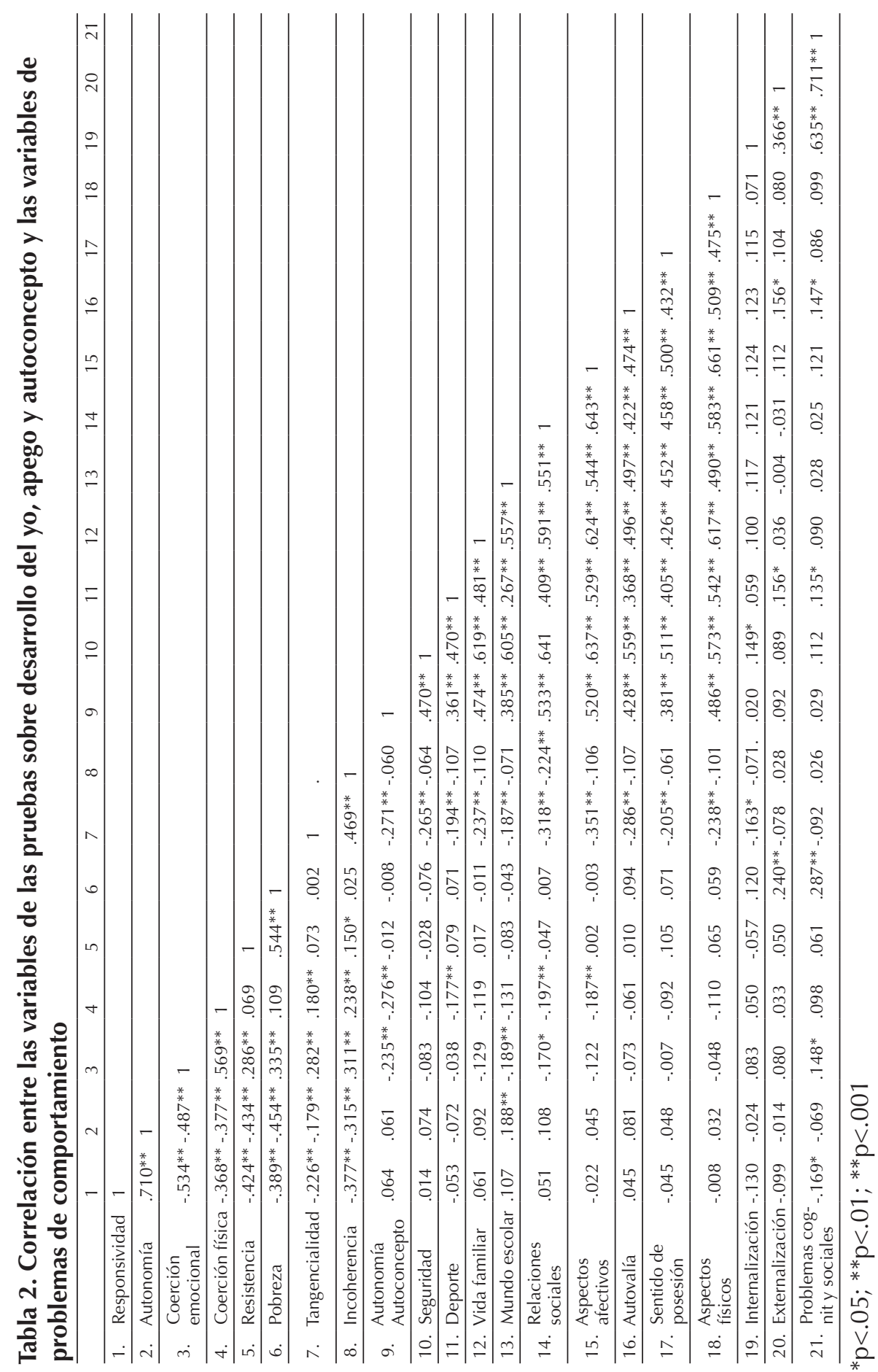


La influencia del apego y el autoconcepto en los problemas de comportamiento de los niños y niñas de familias en desventaja socioeconómica

Alezandra Torres y M. ${ }^{a}$ José Rodrigo

También se encontraron relaciones entre la percepción de autonomía en las narraciones del apego y el ámbito del mundo escolar del autoconcepto, indicando que cuanto más alta era la percepción de autonomía que los menores tenían por parte de sus cuidadores, mayor era el autoconcepto referido al mundo escolar. Asimismo se encontró que la variable coerción emocional correlacionaba con las variables mundo escolar, relaciones sociales y autonomía, indicando que cuanto más coercitivos se percibía a los cuidadores menor era el autoconcepto referido al mundo escolar, las relaciones sociales y la autonomía. Además se encontró que la variable tangencialidad en la estructura de las narrativas, correlacionaba con las siguientes dimensiones del autoconcepto: autonomía, seguridad, deporte, mundo escolar, relaciones sociales, aspectos afectivos, sentido de posesión y autovalía. En todos los casos el signo negativo de esa correlación indicaba que cuanto más tangenciales eran las narraciones que elaboraban los menores, menor era su autoconcepto en cada una de las dimensiones mencionadas. En ese mismo sentido fue la correlación entre la variable incoherencia de las narrativas del apego y el ámbito de las relaciones sociales del autoconcepto, indicando que cuanto más incoherentes eran las narraciones sobre los cuidadores, menor era el autoconcepto relacionado con las relaciones sociales.

\section{Modelos de regresión}

Como se ha señalado, se realizaron una serie de modelos de regresión lineal jerárquica para comprobar el impacto relativo de las variables de apego y autoconcepto sobre los problemas de comportamiento, evaluados por los educadores en el CBCL, como variables dependientes. En todos ellos, en el primer paso se incluyeron como predictoras las variables sociodemográficas de edad y sexo, en el segundo paso las variables de narrativa, en el tercer paso las de autoconcepto, por último, una combinación mixta tanto para las variables de apego como del autoconcepto, resultante de multiplicar cada una de ellas con la variable grupo familiar de procedencia, con el fin de analizar si el impacto de estas variables estaba modulado por el grupo.

Se obtuvieron modelos de regresión significativos con las dimensiones de externalización y problemas cognitivos y sociales (Tabla 3). En el caso de la variable externalización, el primer paso, el valor de la $\mathrm{R}^{2}$ indica que las variables sociodemográficas explican el $7 \%$ de la varian- 
za de la variable dependiente; en el segundo, el valor de la $\mathrm{R}^{2}$ indica que las variables (autonomía y pobreza de las narraciones) de la prueba de narrativa del apego explican el $15 \%$ de la varianza. Lo anterior quiere decir que producir narrativas en las que el cuidador promueve la autonomía, así como presentar narraciones con ausencia de detalles emocionales o pobres, se relaciona con tener comportamientos de externalización, siendo la variable pobreza la que obtuvo mayor peso de las correlaciones semiparciales al cuadrado $\left(r s^{2}=.05\right)$. En el tercer paso, el valor de la $\mathrm{R}^{2}$ de cambio puso de manifiesto un incremento del $4 \%$ de la varianza explicada cuando se incluyeron las variables de autoconcepto, de manera específica se encontró que tener un autoconcepto alto en las relaciones sociales se asocia a tener menos comportamientos de externalización. Por último, el valor de la $\mathrm{R}^{2}$ de cambio puso de manifiesto un incremento del $17 \%$ de la varianza explicada cuando se incluyeron las variables mixtas. Ello indica que las relaciones anteriores están moduladas por la pertenencia de los niños a familias de riesgo o familias normalizadas.

Tabla 3. Regresión lineal jerárquica de las variables del apego, autoconcepto y mixtas sobre lasdimensiones del CBCL (Externalización y Problemas cognitivos y sociales)

\begin{tabular}{|c|c|c|c|c|c|c|}
\hline & \multicolumn{3}{|c|}{ Externalización } & \multicolumn{3}{|c|}{ Problemas Cognitivo-Sociales } \\
\hline & $\beta$ & $\mathrm{R}^{2}$ & $\Delta \mathrm{R}^{2}$ & $\beta$ & $\mathrm{R}^{2}$ & $\Delta \mathrm{R}^{2}$ \\
\hline Paso 1 & & $.07^{* * *}$ & & & .01 & \\
\hline Sexo & $.13^{* * *}$ & & & .10 & & \\
\hline Edad & .08 & & & .06 & & \\
\hline Paso 2 & & $.15^{* * *}$ & $.07^{* * *}$ & & $.12^{* *}$ & $.11^{* *}$ \\
\hline Responsividad & -.04 & & & -.14 & & \\
\hline Autonomia-Narrativa & $.21^{* *}$ & & & $.26^{*}$ & & \\
\hline Coerción emocional & .01 & & & .04 & & \\
\hline Coerción física & .02 & & & .07 & & \\
\hline Resistencia & .03 & & & -.12 & & \\
\hline Pobreza & $.15^{* *}$ & & & $.34 * * *$ & & \\
\hline Tangencialidad & .07 & & & -.13 & & \\
\hline incoherencia & .06 & & & .07 & & \\
\hline Paso 3 & & $.19^{* *}$ & $.04^{* *}$ & & $.15^{*}$ & $.03 *$ \\
\hline Autonomía & .10 & & & $-.50^{*}$ & & \\
\hline Seguridad & .14 & & & .13 & & \\
\hline
\end{tabular}


La influencia del apego y el autoconcepto en los problemas de comportamiento de los niños y niñas de familias en desventaja socioeconómica

Alezandra Torres y M. ${ }^{a}$ José Rodrigo

\begin{tabular}{|c|c|c|c|c|c|c|}
\hline & \multicolumn{3}{|c|}{ Externalización } & \multicolumn{3}{|c|}{ Problemas Cognitivo-Sociales } \\
\hline & $\beta$ & $\mathrm{R}^{2}$ & $\Delta \mathrm{R}^{2}$ & $\beta$ & $R^{2}$ & $\Delta \mathrm{R}^{2}$ \\
\hline Deporte & .08 & & & .08 & & \\
\hline Vida familiar & -.06 & & & .02 & & \\
\hline Mundo escolar & -.06 & & & -.05 & & \\
\hline Relaciones sociales & $-.20 * *$ & & & -.11 & & \\
\hline Aspectos emocionales & .03 & & & .05 & & \\
\hline Autovalía & .08 & & & .05 & & \\
\hline Aspectos físicos & .00 & & & .01 & & \\
\hline Sentimiento posesión & .02 & & & .01 & & \\
\hline Paso 4 & & $.36^{* *}$ & $.17^{* * *}$ & & $.27^{*}$ & $.12^{*}$ \\
\hline Responsividad/grupo & -.03 & & & .15 & & \\
\hline Autono-narra/grupo & .06 & & & -.03 & & \\
\hline Coerc. emoc./grupo & -.03 & & & .05 & & \\
\hline Coerc. física/grupo & .04 & & & -.28 & & \\
\hline Resistencia/grupo & $-.30^{* *}$ & & & -.48 & & \\
\hline Pobreza/grupo & $.36^{* * *}$ & & & $.81^{* *}$ & & \\
\hline Tangencialidad/grupo & .11 & & & .37 & & \\
\hline Incoherencia/grupo & -.00 & & & .15 & & \\
\hline Autonomía/PAl-grupo & .31 & & & -.01 & & \\
\hline Seguridad/grupo & .07 & & & .24 & & \\
\hline Deporte/grupo & .13 & & & .31 & & \\
\hline Vida familiar/grupo & .14 & & & -.04 & & \\
\hline Mundo escolar/grupo & .09 & & & .15 & & \\
\hline Relaciones soc./ grupo & $-.85^{* * *}$ & & & -.34 & & \\
\hline Aspectos emoc./ grupo & .33 & & & $.95^{* *}$ & & \\
\hline Autovalía/ grupo & -.25 & & & -.18 & & \\
\hline Aspectos físic./ grupo & .22 & & & -.27 & & \\
\hline Sentido poses./grupo & .11 & & & -.43 & & \\
\hline
\end{tabular}

Las variables mixtas que obtuvieron un coeficiente de regresión significativo fueron: pobreza-grupo, resistencia-grupo y relaciones socialesgrupo. En la Figura 1, se representa la interacción pobreza-grupo, que resultó ser la variable con mayor peso $\left(r s^{2}=.06\right)$. Según el análisis de varianza realizado, dicha interacción resultó significativa, $F(1,146)=$ $16.33 ; p<.001$. En el grupo de riesgo, la producción de narraciones con alta pobreza, respecto a las de baja pobreza, se relaciona con puntuaciones significativamente más altas de comportamientos externalizados. 


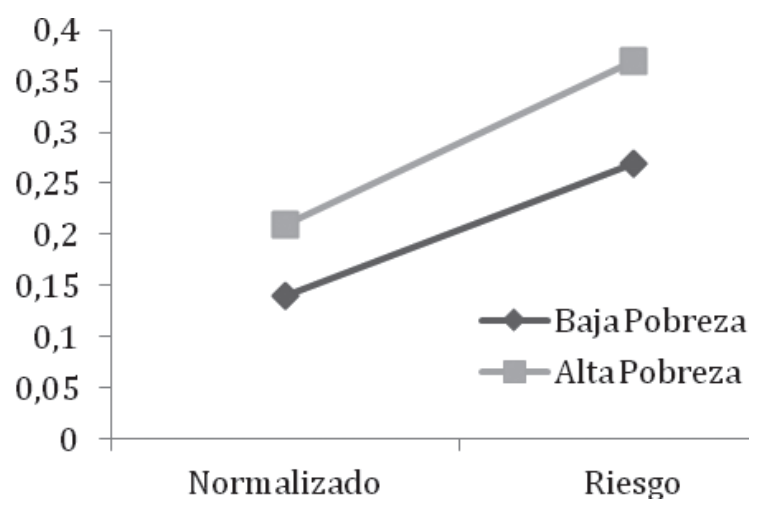

Figura 1. Medias marginales en problemas de externalización según el nivel de pobreza en las narrativas y el grupo familiar

Las interacciones resistencia-grupo y relaciones sociales-grupo no obtuvieron valores significativos en los ANOVAs, pero como tendencia se encontró de una parte que solo en el grupo normalizado la mayor resistencia en la producción de narrativas se asoció a puntuaciones más altas en comportamientos de externalización. De otra parte, en el autoconcepto sobre las relaciones sociales se observa que solo en el grupo de riesgo el tener un autoconcepto menor referido a las relaciones sociales se asoció a puntuaciones más altas en los comportamientos externalizados que el tener un autoconcepto mayor.

En la dimensión problemas cognitivos y sociales de los cuatro pasos de la regresión resultaron significativos los pasos 2 y 4 (Tabla 3). En el segundo paso, el valor de la $\mathrm{R}^{2}$ de cambio indicó un incremento del $11 \%$ de la varianza explicada cuando se incluyeron las variables de la prueba de narrativas. Además encontramos que el producir narrativas en las que se percibe que los cuidadores promueven la autonomía del niño y se caracterizan por una mayor pobreza en su estructura predice una mayor presencia de problemas cognitivo-sociales en el chico/a, siendo la variable pobreza en las narrativas la que obtuvo mayor peso $\left(r s^{2}=.07\right)$. En el cuarto paso, el valor de la $\mathrm{R}^{2}$ de cambio indicó que las variables mixtas añadieron un incremento del $12 \%$ a la varianza explicada. De dichas variables resultó significativa pobreza-grupo con más peso relativo $\left(r s^{2}=\right.$ .03). En la Figura 2, se representa la interacción aspectos emocionalesgrupo, cuyo ANOVA resultó significativo, $F(1,212)=7.75 ; p<.001$. En el grupo de riesgo presentar una mayor emocionalidad ligado al autoconcepto está relacionado con mayores problemas cognitivos y sociales, 
La influencia del apego y el autoconcepto en los problemas de comportamiento de los niños y niñas de familias en desventaja socioeconómica

Alezandra Torres y M. ${ }^{a}$ José Rodrigo

con respecto a aquellos que manifestaron menor emocionalidad, mientras que en el grupo normalizado la diferencia no llega a ser significativa.

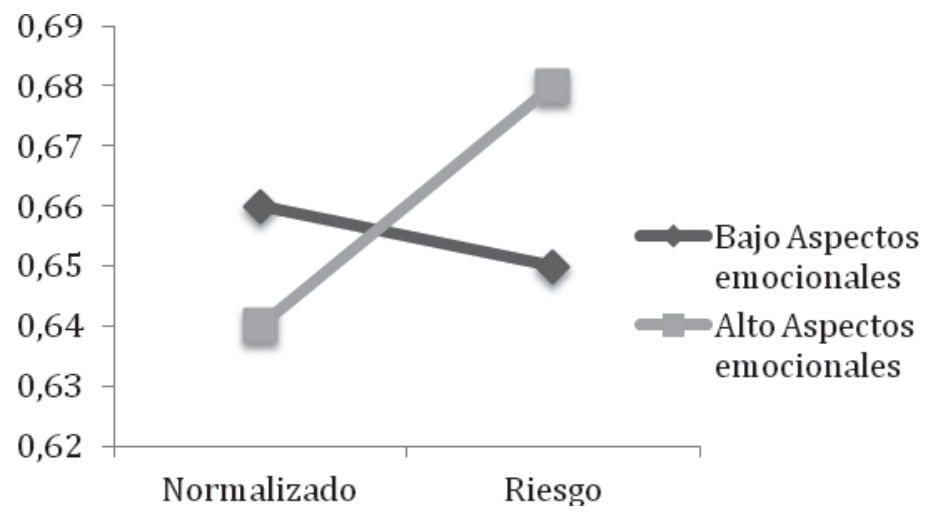

Figura 2. Medias marginales de los problemas cognitivos y sociales según los aspectos emocionales del autoconcepto y el grupo familiar

\section{Discusión}

Las pautas correlacionales y sobre todo los modelos de regresión confirman la relación entre el apego y el autoconcepto y los problemas de comportamiento. En concreto, los modelos de regresión para las variables externalización y problemas cognitivos y sociales, ofrecieron valores explicativos bastante apreciables, el $36 \%$ y el $27 \%$ respectivamente. El valor significativo de la variable sexo, observado en el modelo de externalización indica que son los chicos los que presentaron mayores problemas de comportamientos de tipo externalizado, coincidiendo con los resultados reportados por otros estudios (Achenbach, 1993; Lemos et al., 2002; Sandoval et al., 2006). Los resultados obtenidos confirman que el apego (NICHD Early Child Care Research Network, 2006; Pasco et al., 2011) y el autoncepto y autoestima (Donnellan et al., 2005) son variables que predicen los comportamientos de externalización y agresión principalmente. Sin embargo, son las variables mixtas las que más contribuyen, lo que indica que esa influencia del apego y el autoconcepto está modulada por la pertenencia de los niños/as al grupo familiar de riesgo o normalizado. 
El rasgo del contenido de las narrativas que más se asocia a los problemas de externalización y los cognitivo-sociales es presentar a las figuras de apego favoreciendo los intentos de los chicos/as de hacer las cosas por su propia cuenta. Una posible explicación es que este rasgo es compatible con los comportamientos negligentes en los que los padres y las madres dejan a los chico/as actuar por su cuenta. Por su parte, la pobreza en las narrativas, su simplicidad y falta de detalles de tipo emocional, son un indicio de modelos mentales internos de unas relaciones inadecuadas con las figuras de apego (Shields et al., 2001) y en nuestro caso resulta ser un potente predictor de problemas de comportamiento del tipo externalizado y problemas cognitivos.

En cuanto al autoconcepto, tener un bajo autoconcepto sobre las relaciones sociales, no sentirse capaces de comportamientos autónomos, no tener un buen autoconcepto respecto a la propia familia y tener un buen autoconcepto sobre sus capacidades deportivas se relaciona con los problemas de comportamiento del tipo externalizado y problemas cognitivos. En ese mismo sentido, algunos estudios han encontrado que el autoconcepto y la autoestima se relacionan negativamente con los problemas de ajuste psicosocial y comportamiento antisocial y positivamente con las habilidades sociales y los estilos de vida adecuados sobre todo en la adolescencia (Baumeister et al., 2003; Garaigordobil et al., 2005). Otros estudios han corroborado estos hallazgos en niños de 8 años, en quienes los valores altos de autoconcepto se relacionaron con menores problemas de comportamiento y mayor competencia social tanto en la escuela como en el hogar (Easterbrooks et al., 2000).

El notable impacto añadido de las variables mixtas en la explicación de las variables comportamentales en los modelos de regresión demuestra que el tipo de familia en el que viven los chicos/as está moderando las relaciones entre las relaciones de apego, el autoconcepto y los problemas de comportamiento. Concretamente, la pobreza de las narraciones de apego se relaciona con problemas de externalización y cognitivosociales en el grupo de riesgo, pero no en el grupo normalizado. Sin embargo, la resistencia a la hora de producir las narraciones se relaciona con los problemas de comportamiento de tipo externalizado en los chicos/as del grupo de familias normalizadas pero no en el de riesgo. Por su parte, el bajo autoconcepto en las relaciones sociales y el alto componente emocional ligado al autoconcepto se perfilan como indicadores 
La influencia del apego y el autoconcepto en los problemas de comportamiento de los niños y niñas de familias en desventaja socioeconómica

Alezandra Torres y M. ${ }^{a}$ José Rodrigo

de problemas de externalización y cognitivo-sociales, respectivamente, en el grupo de riesgo pero no en el grupo normalizado.

Los hallazgos más destacados respecto de unas relaciones inadecuadas con las figuras de apego y un bajo autoconcepto en los chico/as del grupo de riesgo psicosocial medio se pueden explicar, porque estas familias suelen darles a sus hijo/as una menor calidad en su trato diario debido a la gran acumulación de estresores psicosociales que vivencian los padres (Rodrigo et al, 2008). Este hecho va a determinar que las prioridades de los padres se modifiquen, disminuyendo tanto su capacidad de atención y evaluación reflexiva de las situaciones educativas, como las posibilidades de un ejercicio parental ajustado a las necesidades derivadas del desarrollo de sus hijo/as.

Por tanto, las representaciones de unas relaciones inadecuadas con las figuras de apego en el grupo de riesgo se explicarían por unas experiencias tempranas poco exitosas en términos de intercambio afectivo, derivadas de una carencia en la accesibilidad física y emocional con las figuras de apego (Brumariu y Kerns, 2010; Weinfield et al., 2008), ya que es con base en dichas experiencias como se construyen los modelos representacionales que más tarde darán lugar a los modelos de funcionamiento interno tal como plantea la teoría del apego. De otra parte, nuestros datos indican que los menores de familias normalizadas tienden a desarrollar representaciones de los cuidadores más adecuadas que probablemente van asociadas a una relación de apego segura, formando un modelo representacional interno más efectivo de sus relaciones con los demás.

De otra parte, como se ha señalado, la calidad de las experiencias tempranas tiene un efecto directo sobre la percepción que los niños construyen de sí mismos, por tanto la noción de autoconcepto depende en gran medida de las experiencias y las relaciones con el entorno afectivo, en el que las personas significativas, como son las primeras figuras de apego, desempeñan un papel importante. Es esperable por tanto que en las familias en situación de riesgo medio, quienes tienen una mayor probabilidad de padecer eventos vitales estresantes, sumado a que en muchos casos cuentan con un repertorio de habilidades parentales reducidas debidas a los bajos niveles educativos, se limite el ejercicio de la parentalidad en aspectos que son fundamentales para favorecer en los hijos/as una noción de autoconcepto adecuada. 
Todo lo anterior confirma que los niño/as de familias en un nivel medio de riesgo psicosocial en la segunda infancia y pre-adolescencia se encuentran con mayor vulnerabilidad de desarrollar problemas de baja autoestima y de comportamiento, según han corroborado algunos estudios realizados con adolescentes en riesgo en el contexto español (Jiménez, 2009; Jiménez et al., 2009). Asimismo, son numerosas las investigaciones que plantean que los menores provenientes de familias en situación de desventaja socio-económica, suelen presentar más problemas conductuales y emocionales que la población de referencia sobre todo en la primera infancia y la adolescencia (Compas, 2004; Kaiser et al., 2000).

Aunque este estudio cuenta con varios informantes y con la posibilidad de acceder a hijo/as de familias en situación de riesgo medio y normalizado perfectamente comparables, en posteriores estudios habrá que incluir otras pruebas sobre el escenario educativo de los menores, así como evaluaciones del comportamiento parental en ambos tipos de familias para contar con más elementos que permitan analizar el impacto de dichas variables en el desarrollo y en particular en aspectos tan trascendentes para la vida adolescente y adulta como son las competencias asociadas al ajuste psicosocial y comportamental.

\section{Conclusiones}

Los resultados de este estudio indican que, en situaciones de desventaja socioeconómica los chico/as en la segunda infancia y pre-adolescencia resultan muy vulnerables a los problemas de externalización y los problemas cognitivo-sociales que pueden acarrear problemas en su desarrollo personal, social y escolar. En especial, hemos visto en este estudio que aspectos como el apego y el autoconcepto están más afectados en estos escolares provenientes de contextos familiares en mayores niveles de vulnerabilidad social, lo que les lleva con mayor facilidad a comportamientos disruptivos en el escenario escolar, lo que puede dificultar su rendimiento escolar. Sin embargo, se debe tener en cuenta que la escuela no siempre es sensible ante esa realidad, produciéndose un gran desencuentro entre la escuela y las familias en situaciones de vulnerabilidad social. En muchas ocasiones este es el sendero que lleva al fracaso escolar, que en parte puede explicarse por 
La influencia del apego y el autoconcepto en los problemas de comportamiento de los niños y niñas de familias en desventaja socioeconómica

Alezandra Torres y M. ${ }^{a}$ José Rodrigo

las mismas situaciones de vulnerabilidad de las familias pero también por la falta de respuesta por parte de la escuela ante estas situaciones. La escuela debe ser ante todo un lugar de intercambio y socialización y para que sea posible una buena educación es imprescindible la comunicación y el intercambio entre las familias y los educadores (García et al 2010).

Estos datos deben además considerarse a la luz de los informes oficiales que señalan una alta tasa de absentismo y abandono escolar en el alumnado proveniente de familias en situación de desventaja socioeconómica (Cifras de educación en España. Estadísticas e indicadores. Edición 2012. Ministerio de Educación). Se observa, por tanto, una continuidad evolutiva muy notable en dicha vulnerabilidad desde la primera infancia, analizada en estudios previos, hasta la pre-adolescencia como se observa en este estudio. Dichos aspectos deberían ser objeto de especial atención en los programas de prevención destinados a la población escolar de modo que éstos promuevan las competencias socio-afectivas para reducir y controlar los efectos adversos sobre el ajuste personal, social y académico de aquellos escolares que viven en familias en desventaja socioeconómica. De no ser así, estos escolares estarán abocados a trayectorias de fracaso escolar que les alejarán definitivamente de las oportunidades de empleo y de realización personal y social.

\section{Referencias bibliográficas}

Achenbach, T.M. (1993). Empirically based taxonomy: How to use syndromes and profile types derived from the CBCL/4-18, TRF and YSR. Burlington, VT: University of Vermont.

Achenbach, T. M., y Edelbrock, C. S. (1983). Manual for the child behavior checklist and revised behavior profile. Burlington: University of Vermont Department of Psychiatry.

Baumeister, R. F., Campbell, J. D., Krueger, J. I. y Vohs, K. D. (2003). Does Self-esteem cause better performance, interpersonal success, happiness, or healthier lifestyle? Psychological Science in the Public Interest, 4, 1.

Bornstein, M.H., Hahn, C-S. y Suwalsky, J.T.D. (2013). Language and internalizing and externalizing behavioral adjustment: Developmental pathways from childhood to adolescence. Development and Psychopathology, 25. 857-878.

Bowlby, J. (1980). Attachment and loss (vol.3). New York: Basic Books.

Bracken, B. A., y Lamprecht, M. S. (2003). Positive self-concept: An equal Opportunity Construct. School Psychology Quarterly, 18, 103-121. 
La influencia del apego y el autoconcepto en los problemas de comportamiento de los niños y niñas de familias en desventaja socioeconómica

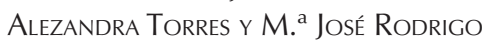

Brumariu, L.E. y Kerns, K.A. (2010). Parent-child attachment and internalizing symptoms in childhood and adolescence: A review of empirical findings and future directions. Developmental and Psychopathology, 22, 177-203.

Burchinal, M., Roberts, J.E., Zeisel, S.A., Hennon, E.A. y Hooper, S. (2006). Parenting and child care factors en early elementary school years. Parenting: Science and practice, $6,79-113$.

Cifras de educación en España. Estadísticas e indicadores. Edición 2012. Ministerio de Educación, Cultura y Deporte. http://www.oei.es/noticias/spip.php?article11289

Compas, B.E., (2004). Processes of risk and resilience during adolescence. En R.M. Lerner y L. Steinberg (Eds). Handbook of adolescent psychology (2a ed., pp. 263-296). New Yersey: John Wiley.

Donnellan, M. B., Trzesniewski, K. H., Robins, R.W., Moffitt, T. E. y Caspi, A. (2005). Low self-esteem is related to aggression, antisocial, behavior and delinquency. Psychological Science, 16, 328-335.

Easterbrooks, M. A., y Abeles, R. (2000). Windows to the self in 8-year olds: Bridge to attachment representation and behavioral adjustment. Attachment \& Human Development, 2, 85-106.

Futh A, O'Connor TG, Matias C, Green J, Scott S. Attachment narratives and behavioral and emotional symptoms in an ethnically diverse, at-risk sample. J Am Acad Child Adolesc Psychiatry. 2008 Jun; 47(6):709-18.

Garaigordobil, M., Durá, A. y Pérez, J. I. (2005). Síntomas psicopatológicos, problemas de conducta y autoconcepto-autoestima: Un estudio con adolescentes de 14 a 17 años. Anuario de Psicología Clínica y de la Salud, 1, 53-63.

García, M. P.; Gomáriz, M.A.; Hernández, M.A. y Parra J. (2010). La comunicación entre la familia y el centro educativo, desde la percepción de los padres y madres de los alumnos. Revista Educatio siglo XXI, 28 (1), 157-188.

Gleason, J.B. y Ratner, N.B. (2008). Development of language ( $7^{\text {th }}$ ed.). Boston: Allyn \& Bacon.

González-Pineda. J.A., Núñez, J.C., González-Pumariega, S., Álvarez, L., Roces, C., García, M., González, P., Cabanach, R. y Valle, A. (2000): Autoconcepto, proceso de atribución causal y metas académicas en niños con y sin dificultades de aprendizaje. Psicothema, 12, 548-556.

Harter, S., Waters, P. y Whitesell, N. R. (1998). Relational self-worth: Differences in perceived worth as a person across interpersonal context among adolescents. Child development, 69,756-766.

Heckman, J. J. (2006). Skill formation and the economics of investing in disadvantaged children. Science, 312, 1900-1902. doi: 10.1126/ science. 1128898

Hill, H.D. y Morris, P. (2008). Welfare policies and very young children: Experimental data on stage-environment fit. Developmental Psychology, 44, 1557-1571. doi 10.1037/a0013913

Hoff, E. (2008). Language development. Boston, MA: Wadsworth

Jiménez, L. (2009). Crecer en contextos familiares en riesgo psicosocial. Tesis doctoral no publicada. Universidad de Sevilla, Sevilla. 
La influencia del apego y el autoconcepto en los problemas de comportamiento de los niños y niñas de familias en desventaja socioeconómica

Alezandra Torres y M. ${ }^{a}$ José Rodrigo

Jiménez, L., Dekovic, M.E., y Hidalgo, M.V. (2009). Adjustment of School-aged children and adolescents growing up in at-risk families: Relationships between family variables and individual, relational and school adjustment. Children and Youth Services Review, 31, 654-661.

Lemos, S. Vallejo, G., y Sandoval, M. (2002). Estructura factorial del Youth Self- Report (YSR). Psicothema, 14, 816-822.

Muris, P., Meesters, C., y Van den Berg, S. (2003). Internalizing and externalizing problems as correlates of self-reported attachment style and perceived parental rearing in normal adolescents. Journal of Child and Family Studies, 12, 171-183.

NICHD, Early Child Care Research Network (2006). Infant-mother attachment classification: Risk and protection in relation to changing maternal caregiving quality. Developmental Psychology, 42, 38-58.

Oppenheim, D. (2003). Children's emotional resolution of MSSB narratives: Relations with child behavior problems and parental psychosocial distress. En R. N. Emde, D.P. Wolf y D. Oppenheim (Eds), Revealing the inner words of young children: The MacArthur Story Stem battery and parent-child narratives (pp. 147-162). Oxford University Press.

Pasco Fearon, R. M. y Belsky, J. (2011). Infant-mother attachment and the growth of externalizing problems across the primary-school years. Journal of Child Psychology and Psychiatry, 52, 782-91.

Rodrigo, M. J., Máiquez, M. L., Martín, J. C. y Byrne, S. (2008). Preservación Familiar. Un enfoque positivo para la intervención con familias. Madrid: Pirámide.

Rodríguez, G., Camacho, J., Rodrigo, M. J., Martín, J. C. y Máiquez, M. L. (2006). La evaluación del riesgo psicosocial en las familias usuarias de los servicios sociales municipales. Psicothema, 18, 200-206.

Sandoval, M., Lemos, S. y Vallejo. G, (2006). Self-reported competences and problems in Spanish adolescents: A normative study of the YSR. Psicothema, 18, 804-809.

Shields, A., Ryan, R.M. y Cicchetti, D. (2001). Narrative representations of caregivers and emotion dysregulation as predictors of maltreated children's rejection by peers. Developmental Psychology, 37, 321-337.

Sroufe, L. A., Carlson, E. A., Levy, A. K. y Egeland, B. (1999). Implications of attachment theory for developmental psychopathology. Development and Psychopathology, 11.1-13.

Villa, A. y Auzmendi, E. (1999). Desarrollo y evaluación del autoconcepto en la edad infantil. Editorial Mensajero. Bilbao España.

Wadsby,M., Svedin,C.G. y Sydsjö, G. (2007). Children of mother at psychosocial risk growing up. A follow up at the age of 16. Journal of Adolescence, 30, 147-164.

Weinfield, N. S., Sroufe, L., Egeland, B. y Carlson, E. (2008). Individual differences in infant-caregiver attachment: Conceptual and empirical aspects of security. En J. Cassidy, \& P. R. Shaver (Eds.), Handbook of attachment: Theory, research, and clinical applications (2nd ed., pp. 78-101). New York: Guilford Press. 